\title{
Creación de un Aula de Formación en Atención Farmacéutica en la Universidad de Salamanca
}

\author{
Ana Martín Suárez¹, Francisco G. López² ${ }^{2}$ Tomás Codesal³ , Elena Valles Martín 4 , \\ Raquel Varas Doval ${ }^{5}$, Antonio Muro ${ }^{6}$ \\ 1. Doctora en Farmacia. Departamento de Farmacia y Tecnología Farmacéutica. Universidad de Salamanca. \\ 2. Doctor en Farmacia. Departamento de Farmacia y Tecnología Farmacéutica. Universidad de Salamanca. \\ 3. Licenciado en Farmacia. Farmacéutico Comunitario en Zamora, Profesor Asociado de Prácticas Tuteladas Universidad de Salamanca. \\ 4. Graduada en Farmacia. Departamento de Química Farmacéutica. Universidad de Salamanca. \\ 5. Licenciada en Farmacia. Farmacéutica Técnica del Departamento de Servicios Asistenciales. Consejo General de Colegios Oficiales de Farmacéuticos. Madrid. \\ 6. Doctor en Medicina. Departamento. de Biología Animal, Ecología, Parasitología, Edafología y Química Agrícola. Universidad de Salamanca.
}

\section{PALABRAS CLAVE}

atención farmacéutica, formación, grado en Farmacia, Universidad

\section{ABREVIATURAS}

AUSAF: Aula de Atención Farmacéutica de la Universidad de Salamanca. CGCOF: Consejo General de Colegios Oficiales de Farmacéuticos. Foro AF-FC: Foro de Atención Farmacéutica en Farmacia Comunitaria.

\section{KEYWORDS}

pharmaceutical care, education, pharmacy degree, universtity

\section{RESUMEN}

Objetivos: Formar un grupo de trabajo, de estudiantes, farmacéuticos y profesores, que lidere actividades formativas y proyectos de investigación en atención farmacéutica desde un aula de la Facultad de Farmacia.

Material y métodos: Fases del proyecto: Organización del espacio físico y obtención de recursos, formación del equipo, identidad y medios de difusión de las actividades, diseño de las líneas de trabajo.

Resultados y discusión: Se ha constituido el equipo responsable: decano de la Facultad, vicedecano responsable de Proyección Farmacéutica, profesor de Atención farmacéutica, titulares de farmacia comunitaria, representante del CGCOF y estudiante de posgrado. Con recursos aportados por la Facultad se ha acondicionado un aula con espacio para impartir talleres a 24 personas, mesas de trabajo y 12 ordenadores. Se ha diseñado un logo y una página en la web de la Facultad. Se ha catalogado una biblioteca, y organizado un sistema de préstamo, con material bibliográfico y software donado por el CGCOF. Se han definido tres líneas de trabajo: impartir talleres de formación en competencias y habilidades asistenciales, impulsar la realización de trabajos Fin de Grado y coordinar un grupo de investigación profesionales-universidad. En conclusión, se ha creado AUSAF, un Aula de Atención Farmacéutica en la Universidad de Salamanca, que pretende la colaboración entre profesionales farmacéuticos y universidad para iniciar proyectos de investigación y adecuar la formación de nuestros graduados a la evolución actual de la profesión, hacia una farmacia asistencial centrada en el paciente. También pretende servir de apoyo a la formación continua de los profesionales.

\section{Creation of a Pharmaceutical Care Training Class in the University of Salamanca}

\section{ABSTRACT}

Objectives: Constitute a working group of students, pharmacists and teachers, to lead training activities and research projects in pharmaceutical care from a classroom of the Faculty of Pharmacy.

Material and methods: Project steps: Physical space organization and resourcing, Team building, Spreading activities, Working lines designed.

Results and discussion: Responsible team: Dean of Faculty, Associate Dean responsible for Pharmaceutical Projection, teacher of Pharmaceutical Care, Community Pharmacist, CGCOF representative and graduate student. Lounge with space for 24 people and 12 computers has been fitted with funds provided by Faculty. A library loan system with bibliographical materials and software donated by CGCOF has been cataloged and organized. It has been defined 3 working lines: workshops provide care skills training, to promote The Final Project of Grade on Pharmaceutical Care and coordinate a research group Professionals-University. In summary, it has been created AUSAF, a classroom of Pharmaceutical Care at the University of Salamanca. AUSAF intends collaboration between pharmacists and University to start research projects and adequate training of our graduates to current developments in the profession, to pharmacy patient-centered care. It also aims to support the continuing education of pharmacists.

Este trabajo ha sido presentado como comunicación al VI Congreso Nacional de Farmacéuticos Comunitarios (Málaga, mayo de 2014), obteniendo el premio a la mejor comunicación en su categoría.

Recibido: $12 / 8 / 2014$

Aceptado: 20-9-2014

Disponible online: 1-12-2014
Financiación: Ninguna ajena.

Conflicto de intereses: Los autores declaran no existir conflicto de intereses en relación con el contenido del presente articulo. Cite este artículo como: Martin A, López FG, Codesal T, Valles E, Varas R, Muro A. Creación de un Aula de Formación en Atención Farmacéutica en la Universidad de Salamanca. Farmacéuticos Comunitarios. 2014 Dec 01; 6(4):40-43. doi:10.5672/FC.2173-9218.(2014/Nol6).004.06 Autora para correspondencia: Ana Martin Suárez (amasu@usal.es).

ISSN 1885-8619 @SEFAC (Sociedad Española de Farmacia Familiar y Comunitaria). Todos los derechos reservados. 


\section{Introducción}

Los grados adaptados al Espacio Europeo de Educación Superior deben conseguir que sus estudiantes adquieran las competencias específicas que les capaciten para desempeñar los perfiles profesionales a los que les da acceso la titulación. Los graduados en Farmacia son profesionales sanitarios que ejercerán mayoritariamente en el ámbito asistencial. La farmacia comunitaria está sufriendo en estos momentos un proceso de cambio orientado a la oferta de servicios profesionales farmacéuticos centrados en el paciente $(1,2)$. A pesar de ello, la formación actual del farmacéutico se sigue basando en unos amplios conocimientos científicos, con una mínima orientación profesional y sin fomentar la labor asistencial.

El objetivo de este trabajo es poner en marcha un aula donde profesores y profesionales implicados en la atención farmacéutica desarrollen proyectos que ayuden a poner en contacto a los estudiantes del Grado en Farmacia con la realidad del ejercicio profesional farmacéutico, favoreciendo el desarrollo de competencias y habilidades asistenciales necesarias para el ejercicio de los servicios de atención farmacéutica. Asimismo, se plantea que el Aula sirva de apoyo a la formación continua de los profesionales farmacéuticos y a la coordinación de proyectos de investigación llevados a cabo en farmacias comunitarias.

Este proyecto no se enmarca en el contexto de ninguna asignatura concreta queriendo implicar a profesores de diferentes áreas e incluso a otros profesionales que integran los equipos multidisciplinares de salud.

\section{Material y métodos}

La puesta en marcha de un aula con objetivos docentes y de investigación en la que participen estudiantes, profesores y profesionales, conlleva una importante dificultad de organización. El proyecto se ha estructurado en 4 fases de distinta índole:

- Organizar el espacio físico del aula y obtención de recursos.

- Formar el equipo responsable.

- Dotar al aula de identidad y medios de difusión de las actividades.

- Diseñar las líneas de trabajo y poner en marcha las primeras actividades.
Las dos primeras etapas se han impulsado desde el decanato de la Facultad, que ha iniciado los primeros contactos para la formación del equipo y cedido un aula en el edificio de la Facultad de Farmacia.

Una vez reunido el equipo responsable del aula, se ha planeado la estrategia para alcanzar los objetivos planteados.

\section{Resultados}

Organización del espacio físico del aula y obtención de recursos

Con recursos aportados por la $\mathrm{Fa}-$ cultad se ha acondicionado un aula con espacio para impartir talleres a 24 personas, mesas de trabajo y 12 ordenadores. Se ha dotado al aula del material bibliográfico y software suficiente para poder documentar y registrar las actividades realizadas. Estos recursos han sido donados por el Consejo General de Colegios Oficiales de Farmacéuticos (CGCOF). Se ha catalogado la biblioteca y organizado un sistema de préstamo.

Para iniciar a los estudiantes en la prestación de servicios asistenciales el aula dispone de una farmacia en el mundo virtual SecondLife ${ }^{\circledR}$ (3). Dicha farmacia se utiliza en la docencia de varias asignaturas del Grado de Farmacia desde los 5 últimos cursos con excelentes resultados (4). En ella es posible simular situaciones que se dan habitualmente en el ejercicio profesional farmacéutico, en un escenario 3D que permite una mayor inmersión que un aula. Los estudiantes deben resolver la situación planteada aplicando los conocimientos adquiridos, lo que permite desarrollar habilidades de comunicación con el paciente. En el siguiente enlace se pueden ver algunos ejemplos de prácticas realizadas con estudiantes de grado y posgrado en este mundo virtual: http://www.youtube. com/watch?v=n521i2Pewqo.

Formación del equipo responsable

Probablemente la fase más importante del proyecto ha sido constituir un equipo capaz de liderar actividades formativas y proyectos de investigación en servicios de atención farmacéutica. Para ello, además de profesores y estudiantes, era necesario implicar a profesionales que ejerzan en este campo.
El equipo ha quedado constituido por el decano Facultad, el vicedecano de Proyección Farmacéutica, la profesora de Atención farmacéutica, una técnica del Área de Servicios Asistenciales del CGCOF, dos farmacéuticos comunitarios y una alumna de posgrado.

Dotación de identidad al aula y medios de difusión de las actividades

Para dar a conocer el aula y que las actividades ofertadas tengan una adecuada difusión, se ha desarrollado una página en la web de la Facultad de Farmacia (figura 1) que se puede visitar en el siguiente enlace: http:// campus.usal.es/ farmacia//AUSAF. Para dotar de identidad al Aula, se necesitaba un nombre y un logo. El nombre finalmente elegido ha sido Aula de Atención Farmacéutica de la Universidad de Salamanca (AUSAF). En la figura 1 también se puede ver el logo diseñado para representar a AUSAF. Se ha intentado conseguir un dibujo muy sencillo que plasmara el concepto de servicios de Atención Farmacéutica.

\section{Diseño de las líneas de trabajo}

Se han definido tres líneas de trabajo donde se encuadrarán las actividades del aula:

- Impartir talleres de formación en competencias y habilidades asistenciales necesarias para el desarrollo de la atención farmacéutica.

- Organizar trabajos Fin de Grado realizados durante las prácticas tuteladas en farmacia comunitaria y tutorar trabajos desde la propia aula.

- Coordinar un grupo de investigadores y profesionales para iniciar proyectos de investigación conjuntos.

En el corto periodo de funcionamiento de AUSAF se han impartido dos talleres con una asistencia de 33 alumnos en total (5). La información, matrícula y organización de los talleres se ha realizado a través de la página web de la Facultad. Al tratarse de las primeras actividades, parecía importante conocer la opinión de los asistentes para identificar las deficiencias y optimizar las próximas ediciones. Al finalizar cada curso, se pidió a los asistentes que cumplimentaran 


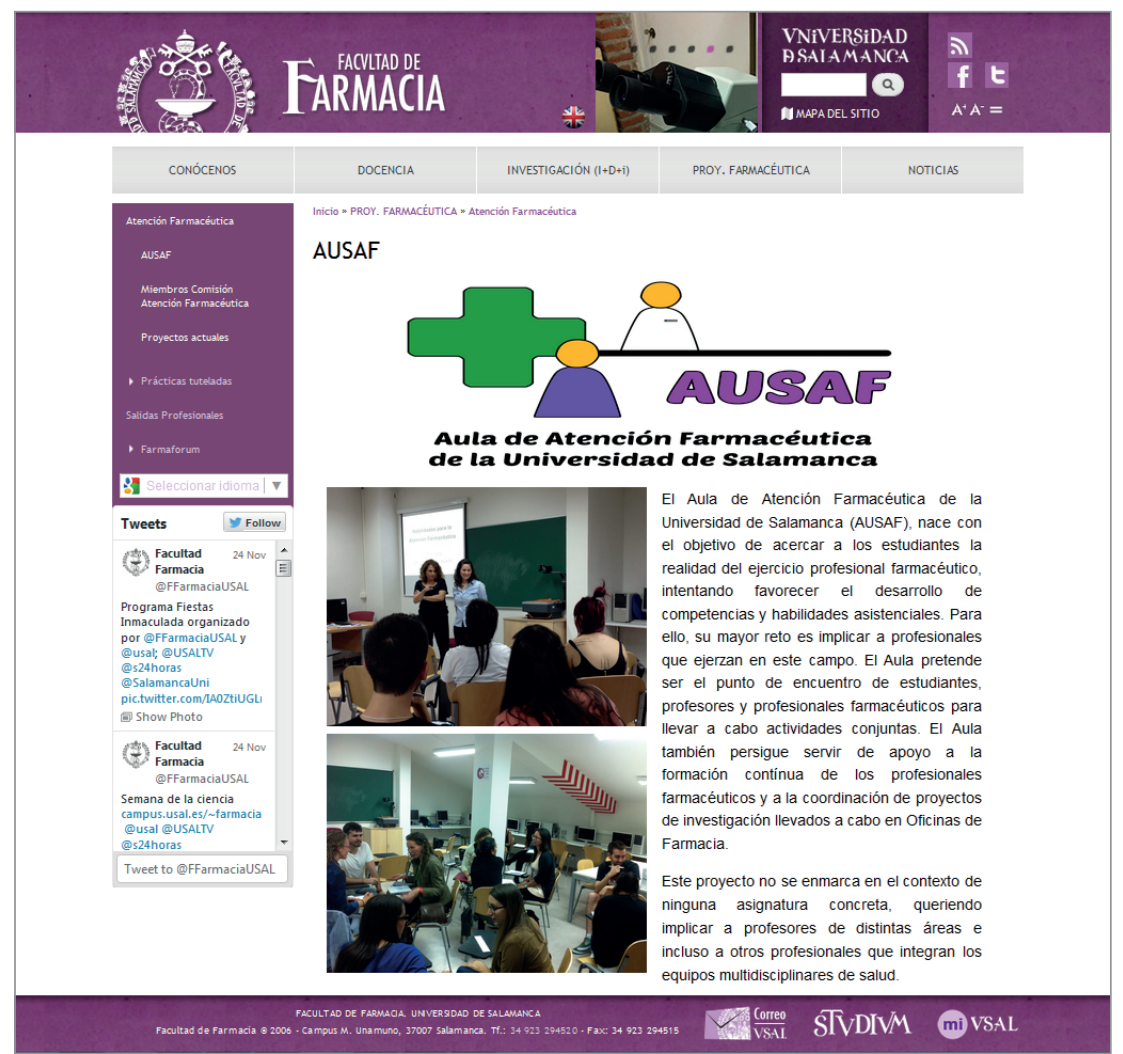

Figura 1 Página de AUSAFen la web de la Facultad de Farmacia

una encuesta online anónima. Los resultados de la satisfacción de los participantes con diferentes aspectos de los talleres impartidos desde AUSAF están recogidos en la tabla 1 .

Inicialmente, se ha seleccionado como tema de trabajo con los alumnos el servicio de Seguimiento farmacoterapéutico a pacientes con diabetes tipo 2. Se han tutorado desde AUSAF cuatro trabajos Fin de Grado sobre el mismo tema, pero con distinta orientación y forma de realizarlos. Dos de los estudiantes han propuesto el servicio de Seguimiento a pacientes habituales de la farmacia comunitaria en la que realizan sus prácticas tuteladas. Los otros dos estudiantes han realizado el seguimiento de pacientes virtuales, pero con situaciones clínicas reales, en la Farmacia virtual disponible en la isla USALPHARMA de SecondLife $(3,4)$.

\section{Discusión}

La iniciativa de crear un aula de atención farmacéutica no es nueva. En otras Facultades de Farmacia se han establecido aulas e incluso farmacias piloto para la práctica de servicios de atención farmacéutica, pero en la mayoría de los casos sólo para la docencia de los estudiantes que cursan esta asignatura. AUSAF quiere abarcar actividades encaminadas a formar a estudiantes de Grado, posgrado e incluso formación continuada de profesionales implicados en los servicios profesionales farmacéuticos, especialmente los relacionados con la atención farmacéutica. Además, pretende ir más allá de la docencia, impulsando la realización de trabajos de investigación en colaboración con otros profesionales sanitarios.

Aunque desde los distintos estamentos (CGCOF, sociedades cientí-

Tabla 1 Puntuación obtenida mediante una encuesta anónima de la satisfacción de los participantes con distintos aspectos de los dos talleres impartidos desde AUSAF

\begin{tabular}{|c|c|c|c|c|}
\hline Calidad del curso & $\begin{array}{c}\text { Calidad del } \\
\text { ponente }\end{array}$ & $\begin{array}{c}\text { Calidad de la } \\
\text { organización }\end{array}$ & $\begin{array}{c}\text { Utilidad de los } \\
\text { conocimientos } \\
\text { adquiridos }\end{array}$ & $\begin{array}{c}\text { Duración del } \\
\text { curso }\end{array}$ \\
\hline $9,2(8-10)^{*}$ & $9,2(8-10)$ & $8,6(8-10)$ & $9,1(8-10)$ & $9,0(7-10)$ \\
\hline
\end{tabular}

* Valor medio (intervalo).

ficas, grupos de investigación...) se están realizando esfuerzos con el fin de conseguir la realización de una práctica profesional asistencial (atención farmacéutica) de calidad, la enseñanza del grado no ha contribuido hasta ahora de forma importante al desarrollo de esta actividad.

Desde AUSAF se pretende conseguir una serie de competencias específicas que, de acuerdo a las conclusiones de la Jornada taller "La Atención Farmacéutica en la Universidad" convocada por el CGCOF y Foro AF-FC (6), se pueden resumir en ser capaz de:

- Evaluar la farmacoterapia de los pacientes en una situación determinada y priorizar las necesidades de intervención.

- Realizar entrevistas clínicas.

- Practicar la comunicación interprofesional y con pacientes.

- Gestionar y liderar el entorno de trabajo.

- Trabajar dentro del sistema sanitario.

En este documento se recomienda incluir en la docencia de atención farmacéutica, metodologías de casos y role-playing, potenciar la formación en técnicas de comunicación y habilidades sociales, realizando:

- Talleres de casos clínicos con participación de otros estudiantes sanitarios.

- Aulas prácticas con pacientes reales y/o simulados.

- Seminarios impartidos por farmacéuticos de hospital, de atención primaria y comunitaria, explicando su práctica profesional.

- Conocimiento del funcionamiento asistencial en centros de salud.

- Estancias en Centros de Información del Medicamento (CIM) en los Colegios Oficiales de Farmacéuticos.

Por todo ello, consideramos que los objetivos de AUSAF están en sintonía con lo que desde la profesión, la organización farmacéutica colegial y las sociedades científicas que conforman Foro AF-FC se está intentando conseguir.

En conclusión, con este proyecto se ha creado un Aula de Atención Farmacéutica en la Facultad de Farmacia (AUSAF) donde estudiantes, profesores y profesionales implicados en la atención farmacéutica se reúnan para desarrollar actividades 
formativas y proyectos de investigación.

Desde AUSAF, en línea con las conclusiones de la jornada-taller de Foro AF-FC, se pretende la colaboración entre los profesionales farmacéuticos y la universidad intentando implicar a todas las estructuras relacionadas (farmacia comunitaria, organización colegial, grupos de investigación, laboratorios farmacéuticos y sociedades científicas) con el fin último de adecuar la formación de nuestros graduados a la evolución actual de la profesión, hacia una farmacia con servicios profesionales farmacéuticos de calidad, centrada en el paciente.

La buena acogida de las primeras actividades desarrolladas por AUSAF nos permite confiar en que la iniciativa ayude a poner en contacto a los estudiantes del Grado de Farmacia con la realidad del ejercicio profesional farmacéutico y sirva de apoyo a la formación continua de los profesionales.

\section{Referencias bibliográficas}

1. Cipolle RJ, Strand L, Morley P. Pharmaceutical Care Practice: The Patient Centered Approach to Medication Management. $3^{\text {a }}$ ed. Mc Graw Hill Medical; 2012. ISBN 13: 978-00-7175638-9.

2. Consejo General de Colegios Oficiales de Farmacéuticos. Foro de Atención Farmacéutica en Farmacia Comunitaria. Guía Práctica para los Servicios de Atención Farmacéutica en la Farmacia Comunitaria. Madrid; 2010. ISBN-13: 978-84-693-1717-4

3. Clavijo Cobaleda E, Díaz Martín J, Martín Suárez A. Farmacia piloto virtual para la docencia en Atención Farmacéutica. VI Congreso Nacional de Atención Farmacéutica. Sevilla; 2009.
(Premio a la mejor comunicación en su sección).

4. Martín Suárez A, Sánchez Vicente D, Martín Izard JF, Clavijo Cobaleda E. Aprendizaje del ejercicio profesional de la Atención Farmacéutica en un mundo virtual. Teoría de la Educación: Educación y Cultura en la Sociedad de la Información. TESI. 2011; 12(4):7187.

5. Pérez Blanco JS, Muro Álvarez A, Cruz Benito J. EducaFarma 2.0 White papers sobre innovación aplicada en el área de las Ciencias Biosanitarias. España. 2014. ISBN 978-84-617-0745-4

6. Foro de Atención Farmacéutica en Farmacia Comunitaria. Jornada-Taller [Internet]. La Atención Farmacéutica en la Universidad. Documento Resumen conclusiones. Madrid 2013. [Acceso 21 julio 2014]. Disponible en: http://www. portalfarma.com/inicio/serviciosprofesionales/forofarmaciacomunitaria/Documents/Conclusiones-Jornada-Universidades-AFFC.pdf 